\title{
The velocity structure of moving magnetic feature pairs around sunspots: support for the U-loop model
}

\author{
J. Zhang ${ }^{1,2}$, S. K. Solanki ${ }^{1}$, J. Woch ${ }^{1}$, and J. Wang ${ }^{2}$ \\ 1 Max-Planck-Institut für Sonnensystemforschung, 37191 Katlenburg-Lindau, Germany \\ e-mail: zjun@ourstar.bao.ac.cn; [solanki; woch]@mps.mpg.de \\ 2 National Astronomical Observatories, Chinese Academy of Sciences, Beijing 100012, China \\ e-mail: wjx@ourstar.bao.ac.cn
}

Received 16 May 2007 / Accepted 14 June 2007

\begin{abstract}
Context. Moving magnetic feature (MMF) pairs are among the most significant fine-scale structures around sunspots. Several models have been proposed to interpret the origin and evolution of MMF pairs. These models provide important clues to understanding MMF pairs.

Aims. We present an analysis of the velocity structure of MMF pairs in order to put further constrains on the MMF models. Methods. Using continuum images, longitudinal magnetograms and Dopplergrams, recorded by the Michelson Doppler Imager (MDI) instrument on the Solar and Heliospheric Observatory (SOHO), we have traced 123 pairs of opposite magnetic polarity moving magnetic features (MMFs) in three active regions NOAA ARs 8375, 0330 and 9575. At the time of observation, AR 8375 was young, AR 0330 mature, and AR 9575 decaying.

Results. The vertical velocity, measured from MDI Dopplergrams for the three active regions, indicates that the elements of MMF pairs with polarity opposite to that of the sunspot support a downflow (Doppler redshift) of around $50-100 \mathrm{~m} \mathrm{~s}^{-1}$. The average Doppler shift difference between negative and positive elements of an MMF pair is about $150 \mathrm{~m} \mathrm{~s}^{-1}$ in AR $8375,100 \mathrm{~m} \mathrm{~s}^{-1}$ in AR 0330, and $20 \mathrm{~m} \mathrm{~s}^{-1}$ in AR 9575. These observational results are in agreement with the model where MMF pairs are part of a U-loop emanating from the sunspot's magnetic canopy. According to this model, the downflow is caused by the Evershed flow returning below the solar surface. For AR 8375, the horizontal velocity of MMFs ranges from $0.1 \mathrm{~km} \mathrm{~s}^{-1}$ to $0.7 \mathrm{~km} \mathrm{~s}^{-1}$, and on average, the velocity of an MMF pair decreases significantly (from $0.6 \mathrm{~km} \mathrm{~s}^{-1}$ to $0.35 \mathrm{~km} \mathrm{~s}^{-1}$ ) with increasing distance from the MMF's birth place. In contrast, the decrease of the average velocity is far less obvious from $0.5 \mathrm{~km} \mathrm{~s}^{-1}$ to $0.4 \mathrm{~km} \mathrm{~s}^{-1}$ with increasing distance from the sunspot. This result suggests that the change in MMF flow speed does not reflect the radial structure of the moat flow, but rather is intrinsic to the evolution of the MMF pairs. This result is also in agreement with the U-loop model of MMF pairs. We also find that properties of MMF pairs, most strikingly the lifetime, depend on the evolution stages of the parent sunspot. The mean lifetimes of MMF pairs in ARs 9575 and 0330 are $0.7 \mathrm{~h}$ and $1.6 \mathrm{~h}$, respectively, which is considerably shorter than the $4 \mathrm{~h}$ lifetime previously found for AR 8375 .
\end{abstract}

Key words. Sun: chromosphere - Sun: magnetic fields - Sun: sunspots

\section{Introduction}

Moving magnetic features (MMFs) are small magnetic structures that move away from a sunspot to the periphery of the surrounding moat (Vrabec 1971; Harvey \& Harvey 1973; Muller \& Mena 1987; Brickhouse \& LaBonta 1988; Lee 1992; Zhang et al. 2003; Hagenaar \& Shine 2005). These MMFs have been classified into three types and their properties have been summarized by Shine \& Title (2001; see also Weiss et al. 2004, for a review). Type I MMFs consist of bipolar pairs of magnetic elements. The bipolar pairs move jointly outward across the moat at speeds of $0.5-1 \mathrm{~km} \mathrm{~s}^{-1}$. They usually first appear just outside the sunspot along a radial line extending from a dark penumbral filament, although some MMFs originate inside penumbrae (Sainz Dalda \& Martínez Pillet 2005). Type II MMFs are single magnetic elements with the same polarity as the sunspot, and mainly originate inside penumbrae (see Ravindra 2006; Zhang et al. 2007), moving outward across the moat at speeds similar to that of type I MMFs, while type III MMFs are single magnetic elements with polarity opposite to that of the sunspot, moving outward at significantly higher speeds of $2-3 \mathrm{~km} \mathrm{~s}^{-1}$.
Harvey \& Harvey (1973) proposed a model in which magnetic flux is removed from the sunspot at the photospheric level. In this model flux tubes form a sea serpent and MMFs are the intersections of these flux tubes with the solar surface. An alternative possibility was suggested by Wilson (1973, 1986; cf. Spruit et al. 1987). In his model, a thin magnetic flux tube is detached from the main flux of the sunspot well below the surface. The detached tube moves turbulently to the surface, developing twists and kinks, which are seen as MMFs once it reaches the solar surface. Also in this model a structure similar to a sea serpent can be formed. Finally, Ryutova et al. (1998) have modelled MMF pairs as $\Omega$ loops emerging from below. They propose that these loops are kinks of a horizontal flux tube lying below the surface. They model the propagating kinks as a solitary wave.

Using Big Bear Solar Observatory, Yurchyshyn et al. (2001) studied the longitudinal magnetic fields of $28 \mathrm{MMF}$ pairs, associated with two large sunspots. They find that MMFs are not randomly oriented. The magnetic element having the same polarity as the sunspot is located further from the sunspot than the opposite polarity element. Furthermore, they find a correlation between the orientation of the MMF bipoles and the twist 
of the sunspot superpenumbra, as deduced from $\mathrm{H} \alpha$ images. Zhang et al. (2003) confirmed the results of Yurchyshyn et al. (2001) for a larger sample of MMF pairs, and deduced further systematics of MMFs properties. MMFs tend to cluster at particular azimuths around the parent sunspot and move approximately radially outward from sunspots at an average speed of $0.45 \mathrm{~km} \mathrm{~s}^{-1}$. Their motion is deflected towards large concentrations of magnetic flux of opposite polarity to that of the parent sunspot. Zhang et al. argued that these and other observations are best reproduced by a model in which MMFs are the intersections of U-loops, produced by localized dips of the magnetic canopy surrounding sunspots, with the solar surface.

Inside and around sunspots, many flows have been observed, which may affect the velocity structure of MMFs (Solanki 2003). Such flows are: 1. Evershed flow (Evershed 1909), a predominantly radial horizontal outflow seen in the penumbra (Muller 1992; Thomas 1994; see also Borrero et al. 2005). Flow velocities of several $\mathrm{km} \mathrm{s}^{-1}$ (Bumba 1960; Wiehr 1995; Schlichenmaier \& Schmidt 2000) and even supersonic values (del Toro Iniesta et al. 2001) have been reported in connection with the Evershed flow; 2. moat flows, radial outflows around decaying sunspots (Sheeley 1969; 1972); 3. downflows near the outer penumbral border and upflows near the inner penumbral border (Westendorp Plaza et al. 1997, 2001; Hirzberger \& Kneer 2001; cf. Tritschler et al. 2004).

In this paper we mainly study the horizontal and Doppler velocity of MMF pairs around three sunspots in active regions NOAA 8375, 9575 and 0330 . The velocity structure of MMFs provides additional constraints that a successful model must satisfy. One aim of the present paper is to test to what extent the model proposed by Zhang et al. (2003) is able to reproduce these additional observations. We also consider whether the properties of the MMFs depend on the evolution stages of the sunspot.

\section{Observations and Analysis}

We combine magnetic field and Doppler velocity observations carried out by the Michelson Doppler Imager, MDI (Scherrer et al. 1995) on the Solar and Heliospheric Observatory (SOHO). MDI was employed in the high-resolution mode (0.625 arcsec per CCD pixel and a 1 min cadence). Observations of three active regions, NOAA ARs 8375, 0330 and 9575, are analyzed. At the time of observations, the three active regions were at three different evolutional stages. AR 8537 was young and still exhibited some flux emergence, AR 0330 was regular and mature, and AR 9575 was also regular but decaying. All three active regions were located near the central meridian (N18W06 for AR 8375, N10E05 for AR 9575 and N09W01 for AR 0330). Each active region has a relatively large compact leading sunspot of positive polarity and an extended negative polarity region. Figure 1 shows MDI continuum images (left) of ARs 8375 (top row), AR 9575 (middle row) and AR 0330 (bottom row), corresponding MDI longitudinal magnetograms (middle), as well as corresponding MDI Dopplergrams (right).

To eliminate the Doppler signal caused by the 5 -min oscillations, we averaged over five successive Dopplergrams. Thus, Dopplergrams with a cadence of 5 min were analyzed. We identified $42 \mathrm{MMF}$ pairs in AR 8375 during $40 \mathrm{~h}$ of observation time (from 1998 November 23 18:53 UT to 25 10:51 UT), 55 MMF pairs in AR 9575 during $11 \mathrm{~h}$ (from 2001 August 16 19:38 UT to 17 06:48 UT) and $12 \mathrm{~h}$ (from 2001 August 17 18:09 UT to 18 06:25 UT), respectively, and $26 \mathrm{MMF}$ pairs in AR 0330 during $10 \mathrm{~h}$ (from 2003 April 9 14:05 UT to $1000: 48 \mathrm{UT})$.
The MMF pairs were identified by visually scanning successive magnetograms. For a feature to be selected as an MMF, we required it to appear in at least 10 magnetograms. We selected only well-isolated MMF pairs. This may bias our selection towards tighter pairs. The studied MMFs were located around the leading sunspots.

Zhang et al. (2003) determined a set of parameters for each pair of MMFs identified in two young active regions NOAA ARs 8375 and 9236 . Here we extend their study by analysing a mature active region (AR 0330), and a decaying one (AR 9575). This will help to disentangle the properties of MMFs in active regions at different stages of their evolution. In addition, we also investigate physical parameters not considered by Zhang et al. Besides analysing the location of first appearance and the lifetime of MMF pairs, we concentrate on the velocity structure of MMF pairs.

Location relative to the sunspot and horizontal velocity of MMF pairs is determined in co-aligned magnetograms. We determine the time an MMF pair firstly appears, record the position " $\mathrm{P}_{0}$ " of the point on the penumbral boundary, which is nearest to the MMF pair, and the barycentral position " $\mathrm{P}_{b}$ " of each element belonging to the MMF pair. Then we track the MMF pair while it moves outward until it disappears, and record each one hour the barycentral position. From this barycentral position we determine the distance to " $\mathrm{P}_{0}$ " and the relative distance from " $\mathrm{P}_{\mathrm{b}}$ ". The velocity is determined by measuring the distance travelled by each MMF element in the given time interval. Of course it is possible that the penumbral boundary evolves during the lifetime of an MMF pair. This can introduce some scatter into, e.g., figures involving the distance from the penumbral boundary, but should not bias the results unduly since we consider MMFs located all around the sunspot.

\section{Location of first appearance and lifetime of MMFs in ARs 8375, 0330 and 9575}

For young active regions, the majority of MMF pairs first appears at a distance of 1000 to $7000 \mathrm{~km}$ from the outer boundary of sunspots. The mean distance at first appearance is $4500 \mathrm{~km}$, with standard deviation of $3800 \mathrm{~km}$. The mean lifetime of MMFs is around $4 \mathrm{~h}$ (Zhang et al. 2003), with standard deviation of $2.1 \mathrm{~h}$.

Figure 2 shows the distribution of the distance to the sunspot penumbral boundary at first appearance of $123 \mathrm{MMF}$ pairs, identified in the young active region (AR 8375), the mature one (AR 0330), and the decaying one (AR 9575). In order to compare to the results of Zhang et al., we applied the same analysis approach. Negative distance values mean that the corresponding MMF pairs appear inside the penumbrae (i.e. within the three closed dotted curves in the continuum images of ARs 8375, 0330 and 9575 in Fig. 1). For mature and decaying active regions, 12 out of the 81 MMF pairs were first seen within the penumbral area, although we cannot rule out that some were missed against the relatively strong penumbral signal. The mean distance at first appearance of the 69 MMF pairs first observed outside the penumbra is $3100 \mathrm{~km}$ with a standard deviation of a $2300 \mathrm{~km}$. This distance is somewhat shorter than the mean distance of $4500 \mathrm{~km}$ found in young active regions (see also Zhang et al. 2003).

By tracking MMF pairs in the three active regions from birth to death, we have determined their lifetimes. Figure 3 shows the lifetime distribution of the 123 MMF pairs. For the MMFs in AR 9575, the lifetime ranges from 0.2 to $2.2 \mathrm{~h}$, with the peak 


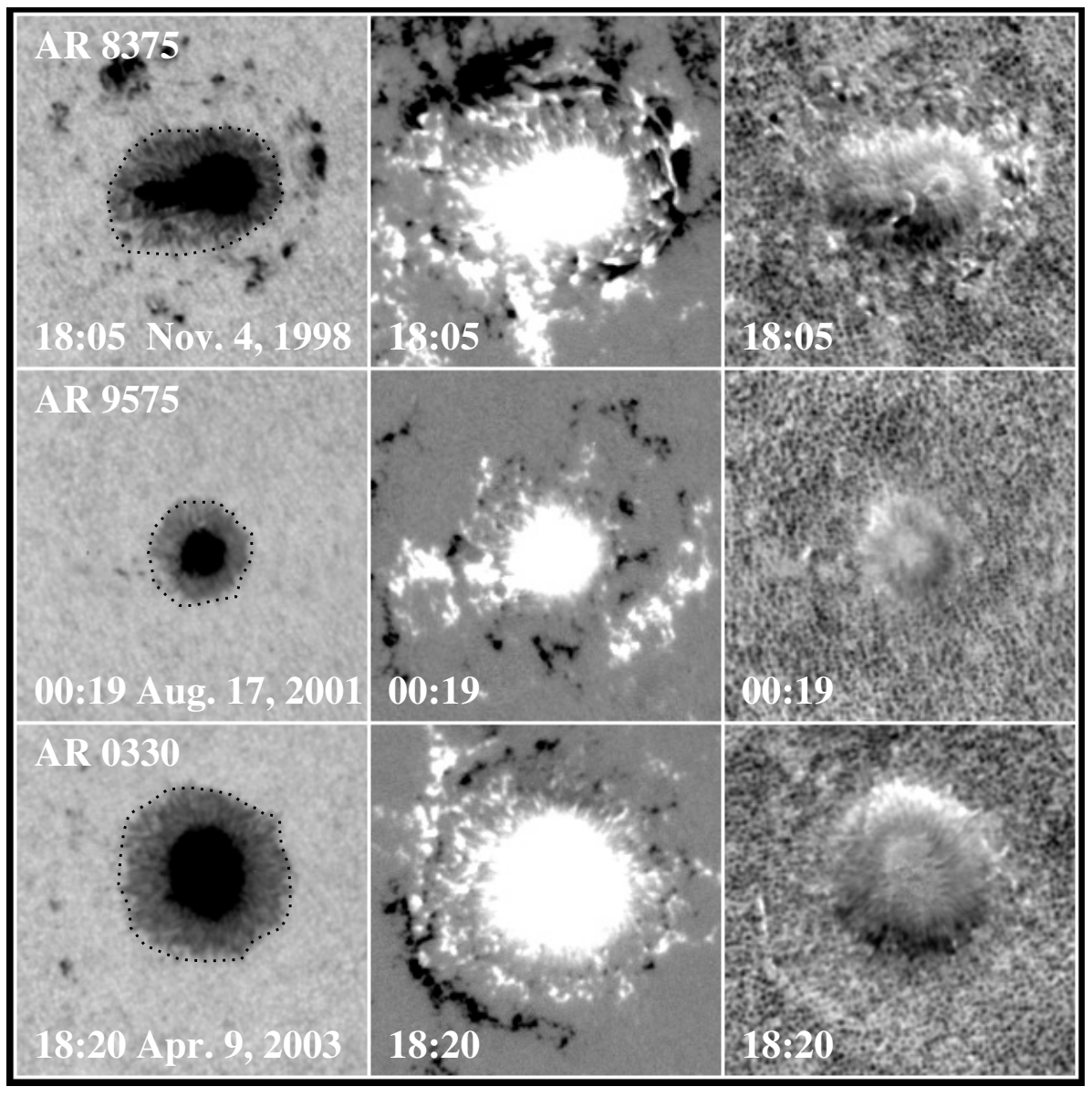

Fig. 1. Left: MDI continuum images of NOAA AR 8375 (top row), NOAA AR 9575 (middle row) and NOAA AR 0330 (bottom row); middle: corresponding MDI longitudinal magnetograms; right: corresponding MDI Dopplergrams. The dotted curves in the continuum images outline the boundary of the penumbrae.

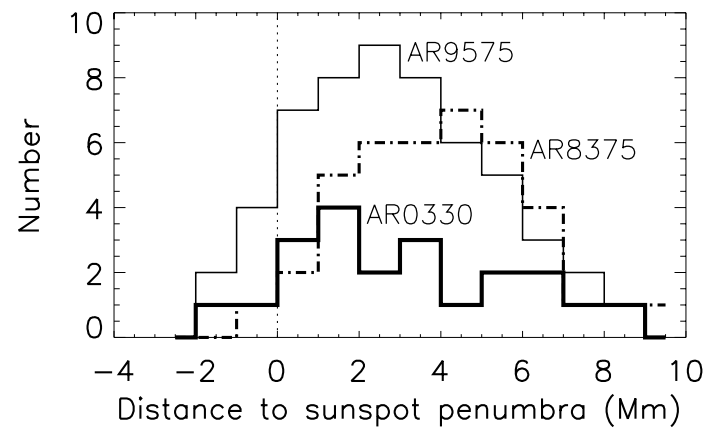

Fig. 2. Histogram of the distance of first appearance of MMF pairs from the sunspot boundary of the three active regions ARs 9575, 0330 and 8375.

of the distribution close to $0.6 \mathrm{~h}$. The average lifetime is $0.7 \mathrm{~h}$ with standard deviation of $0.3 \mathrm{~h}$. For the MMFs in AR 0330, the lifetime ranges from 0.5 to $2.7 \mathrm{~h}$, and the mean lifetime is $1.6 \mathrm{~h}$ with standard deviation of $0.6 \mathrm{~h}$, which is longer than that in the decaying active region, AR 9575. The lifetimes for these two ARs are significantly shorter than the $4 \mathrm{~h}$ found for the young active region AR 8375 (e.g. Zhang et al. 2003).

\section{The velocity structure of MMF pairs}

\subsection{Doppler velocity}

An interesting parameter of MMF pairs is the Doppler shift inside MMF elements. Figure 4 presents examples of two individual elements of an MMF pair. It shows MDI line-of-sight

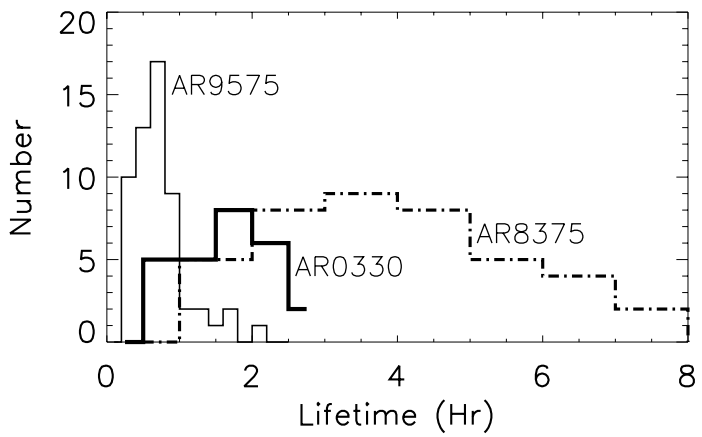

Fig. 3. Histogram of MMF lifetimes in the three active regions ARs 9575, 0330 and 8375.

magnetograms (left) and corresponding MDI Dopplergrams (right) in the northern region of the sunspot of the young AR 8375 on Nov. 4, 1998. The rectangular box on the magnetogram at 01:27 UT marks an MMF pair. The time series of magnetograms and Dopplergrams in the lower frames exemplify the horizontal motion of the MMF pairs within a 30 min time interval as well as the distribution and evolution of downflows (white patches), respectively, upflows (black patches). The outward motion of the MMF is clearly visible. Furthermore, the negative element of the MMF (i.e. the polarity opposite to that of the parent sunspot) shows significant and temporally stable downflow. Figure 5 shows MDI magnetograms and corresponding MDI Dopplergrams of the north-western region of the sunspot in the mature AR 0330. Again, the negative element of the MMF pair shows a downflow and the magnitude of the flow is stable with time. 


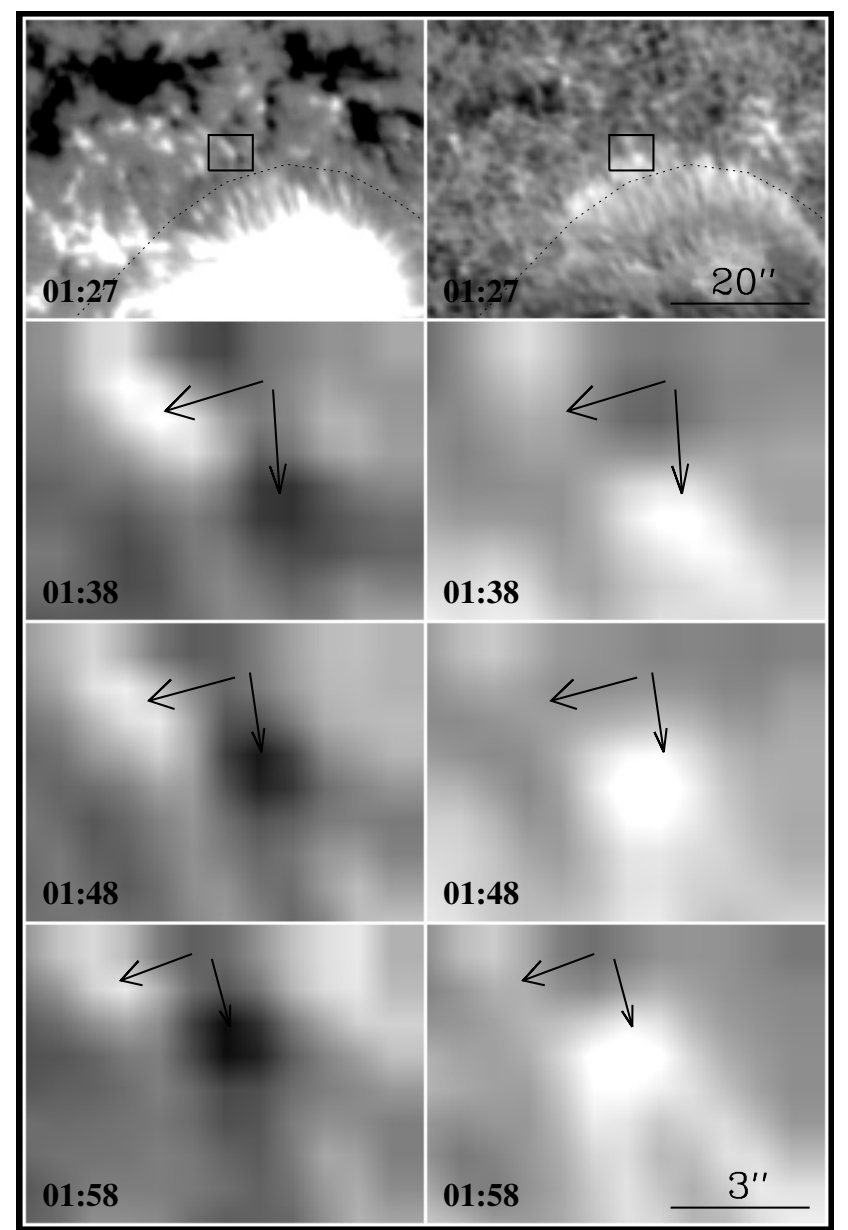

Fig. 4. Left: SOHO/MDI longitudinal magnetograms showing the northern part of the sunspot of AR NOAA 8375 on Nov. 4, 1998. A box at 01:27 UT outlines the field-of view of the following magnetograms. The arrows denote an MMF pair; right: corresponding MDI Dopplergrams. White patches show downflow, and black patches, upflows. The arrows point to the location of the MMF pair.

The left column of Fig. 6 displays the histogram of the Doppler shift of the individual MMFs, identified from the three active regions, with the thick line referring to MMF elements of positive polarity (i.e. the same as the sunspot), the thin line to negative elements. The figure also shows the difference of Doppler shift between the negative and positive elements. For the young active region NOAA AR 8375, the Doppler shift of elements of both polarity ranges from $-350 \mathrm{~m} \mathrm{~s}^{-1}$ (blueshift) to $550 \mathrm{~m} \mathrm{~s}^{-1}$ (redshift). However, the distribution of the negative polarity is shifted towards positive velocities. For the elements of positive polarity, the peak is located near $-50 \mathrm{~m} \mathrm{~s}^{-1}$, for the negative polarity element at $+100 \mathrm{~m} \mathrm{~s}^{-1}$. The average difference of the Doppler shift between negative and positive elements of an MMF pair is $150 \mathrm{~m} \mathrm{~s}^{-1}$, as shown in the top-right frame of Fig. 6, and the standard deviation of the Doppler shift of an MMF element is about $55 \mathrm{~m} \mathrm{~s}^{-1}$. This implies that plasma in the negative element moves downward relative to that in the positive element, and the difference is significant. For the mature active region AR 0330, the Doppler shift of MMFs ranges from $-300 \mathrm{~m} \mathrm{~s}^{-1}$ to $550 \mathrm{~m} \mathrm{~s}^{-1}$. The peak is located near $0 \mathrm{~m} \mathrm{~s}^{-1}$ for positive polarity elements, and $100 \mathrm{~m} \mathrm{~s}^{-1}$ for negative polarity elements. The difference is $100 \mathrm{~m} \mathrm{~s}^{-1}$, with standard deviation of roughly $36 \mathrm{~m} \mathrm{~s}^{-1}$. Again, plasma in the element closer to the sunspot moves downward relative to that in the more remote

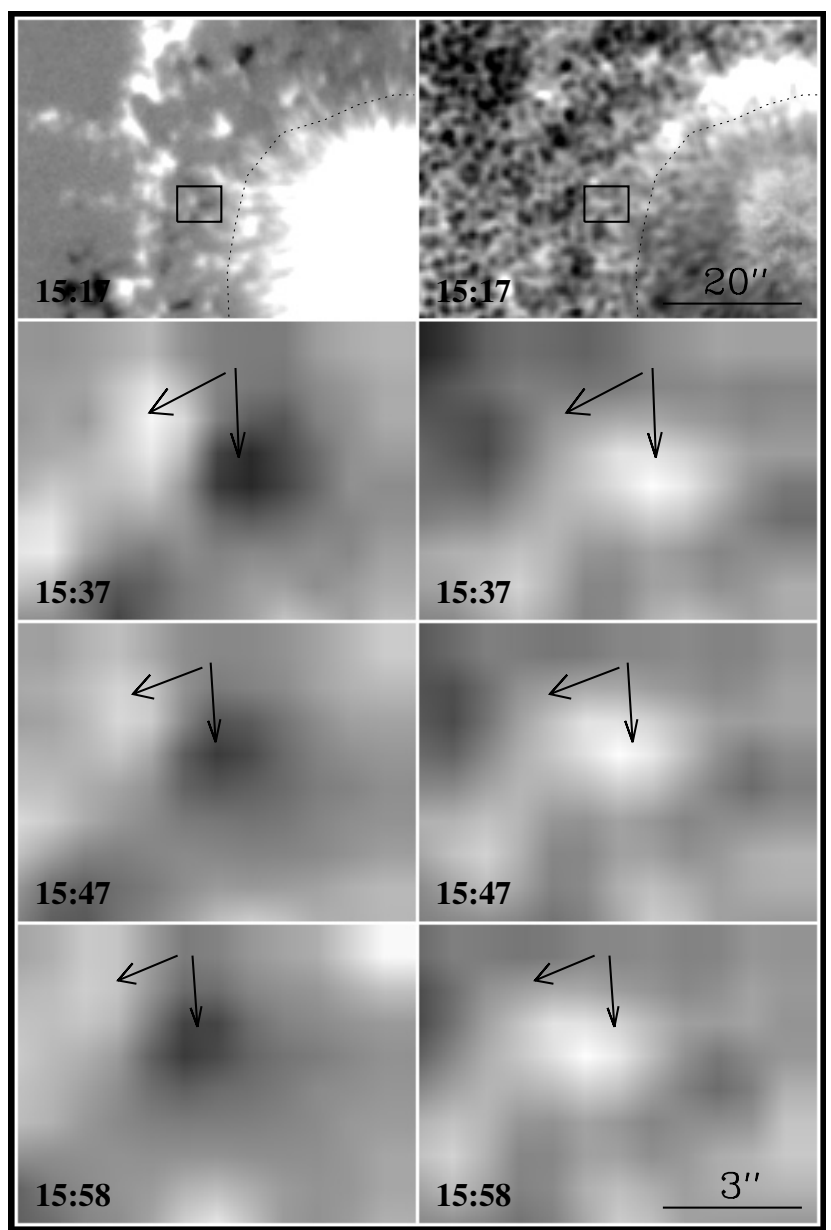

Fig. 5. Similar to Fig. 4 but for AR 0330. Left: MDI longitudinal magnetograms showing the north-western part of the sunspot; right: corresponding MDI Dopplergrams.

element. The Doppler shift of MMFs in the decaying active region (AR 9575) ranges from $-170 \mathrm{~m} \mathrm{~s}^{-1}$ to $280 \mathrm{~m} \mathrm{~s}^{-1}$, and the peak is located at $20 \mathrm{~m} \mathrm{~s}^{-1}$ and $40 \mathrm{~m} \mathrm{~s}^{-1}$ for positive and negative polarity elements, respectively. There is a smaller difference $\left(20 \mathrm{~m} \mathrm{~s}^{-1}\right)$ of the Doppler shifts between the negative and positive elements, compared to those of the young and mature active regions. This difference is below the $1 \sigma$ level $\left(24 \mathrm{~m} \mathrm{~s}^{-1}\right)$ and is not significant.

We have also studied the evolution with time of the difference in Doppler shifts between positive and negative elements. Generally, for a given MMF pair the difference of the Doppler shifts between the negative and positive elements is remarkably stable as a function of time. E.g. for AR 8375, the difference at first appearance is $157 \mathrm{~m} \mathrm{~s}^{-1}$, and at last detection, $151 \mathrm{~m} \mathrm{~s}^{-1}$

The zero level for the line-of-sight velocity in Fig. 6 was set by taking the average Doppler shift of a quiet-Sun region at the same longitude and setting this to zero. This implies that the zero level corresponds to a small redshift (typically $200-300 \mathrm{~m} \mathrm{~s}^{-1}$ for photospheric lines of neutral metals), since the granular blue shift of the Ni I line has not been removed. This means that the downflow in the negative polarity MMFs is larger than indicated by this figure. 

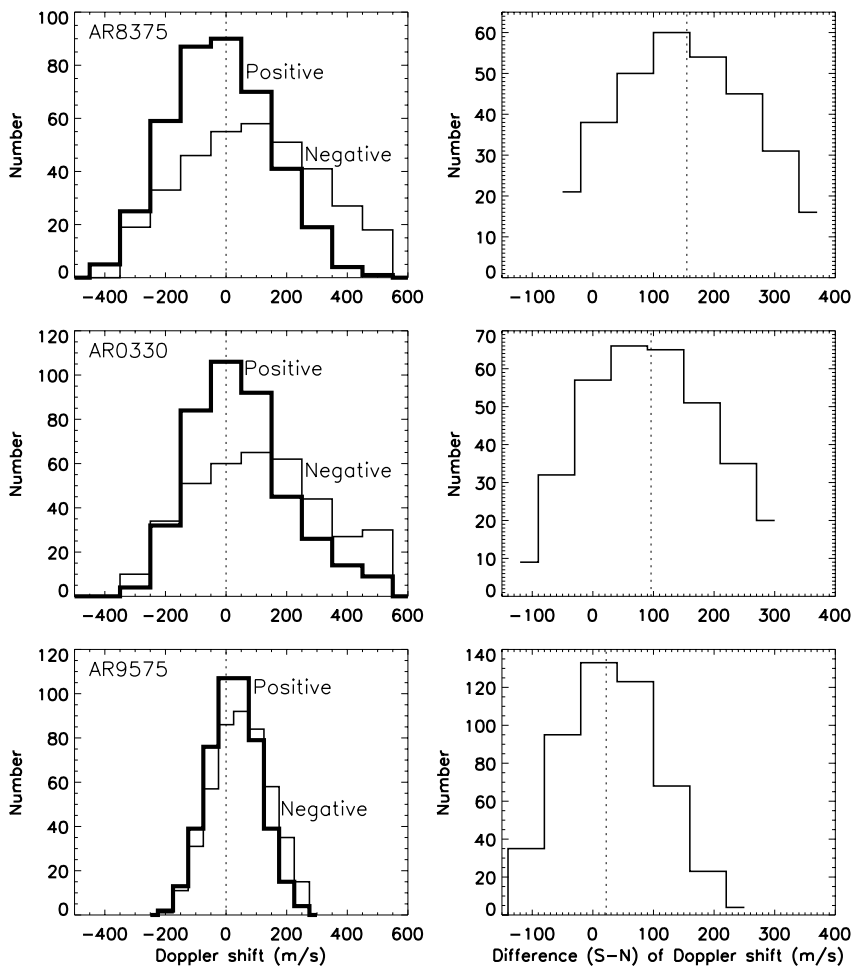

Fig. 6. Left column: histogram showing the Doppler shift of the individual MMFs in the three active regions; right column: histogram showing the difference of the Doppler shift between the negative and positive MMF elements in the three active region.

\subsection{Evolution of the horizontal velocity}

In general, MMF pairs move roughly radially away from the center of the parent sunspot. Zhang et al. (2003) reported that the average horizontal velocity is $0.45 \mathrm{~km} \mathrm{~s}^{-1}$ in the young active region AR 8375. Here we analyse the evolution of the horizontal velocity with distance from the penumbral boundary and from their birth place. Due to the short lifetime of MMFs in ARs 9575 and 0330, these MMFs move only a short distance before disappearing, so that it is difficult to measure changes in their horizontal speed. We therefore only analysed the velocity of the 42 MMF pairs in AR 8375. Figure 7 shows the relationship between horizontal velocity and distance to the sunspot penumbra of AR 8375, separately for positive (top) and negative (bottom) elements. The horizontal velocity decreases from an average value of $0.5 \mathrm{~km} \mathrm{~s}^{-1}$ to $0.4 \mathrm{~km} \mathrm{~s}^{-1}$, as the distance to the sunspot penumbra increases from $2000 \mathrm{~km}$ to $12000 \mathrm{~km}$. However, the scatter is large and the trend not entirely clear. This is reflected by the low absolute values of the correlation coefficient (given in the figure).

Figure 8 displays the horizontal velocity of MMF elements versus relative distance from MMF birth place. The velocity decreases from $\sim 0.6 \mathrm{~km} \mathrm{~s}^{-1}$ to $0.35 \mathrm{~km} \mathrm{~s}^{-1}$, with a strongly increased anti-correlation between velocity and relative distance from MMF birth place. This means that usually MMF elements have a higher radial velocity at their first appearance, but slow down while moving from the sunspot to the periphery of the surrounding moat.

The main source of error of velocity determination of MMF elements is due to uncertainty in the MMF position. A position error of one pixel introduces an error of the velocity of (one pixel)/(time interval). As the size of one pixel is about 0.6 arcsec and the time interval is about $1 \mathrm{~h}$, the error in velocity
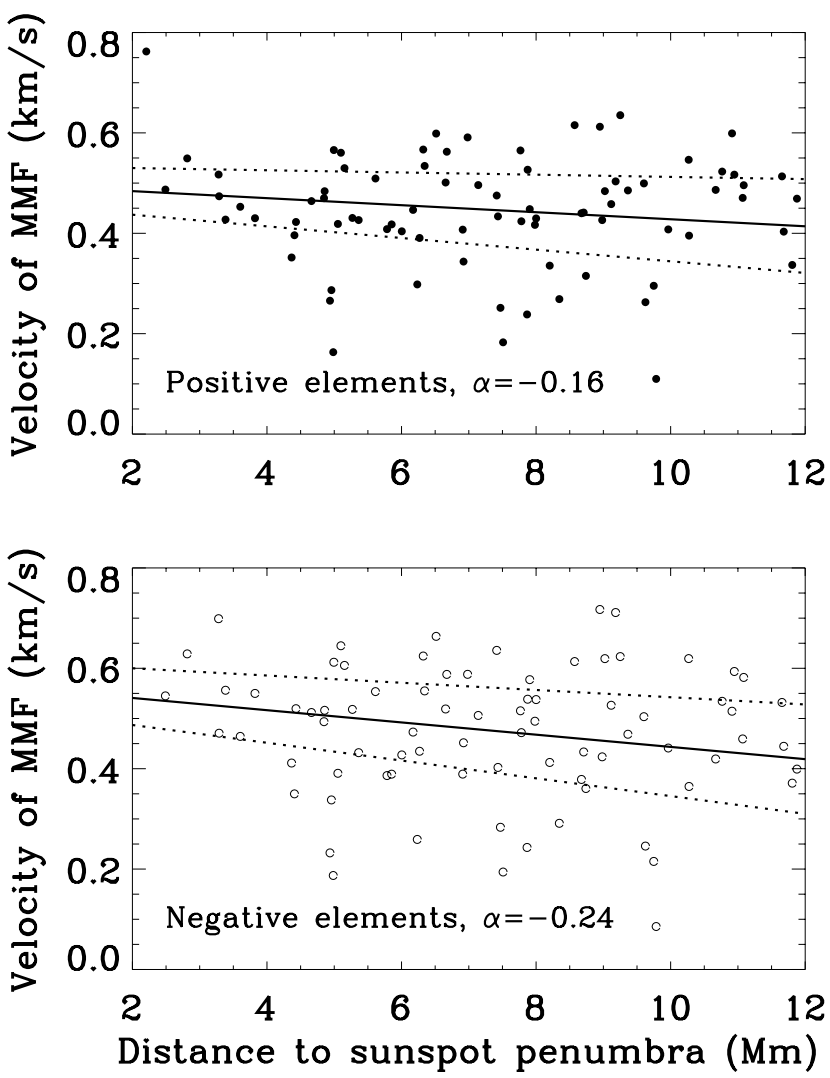

Fig. 7. The relationship between horizontal velocity and distance to the sunspot penumbra of AR NOAA 8375. Upper panel: horizontal velocity of the positive elements of MMF pairs; lower panel: horizontal velocity of the negative elements. In each panel, the solid line represents a linear regression, with the $1 \sigma$ uncertainty of the velocity outlined by two dotted lines, $\alpha$ represents the correlation coefficient.

becomes $0.1 \mathrm{~km} \mathrm{~s}^{-1}$, which may explain a part of the scatter in Figs. 7 and 8.

Note that the MMF velocity found by us, at least during the later stages of an MMF pair's life, is similar to those of intranetwork magnetic elements (Zhang et al. 1998), which are thought to be dragged along by the supergranular flow. The initially higher speed of the MMFs may have two causes: either the moat flow is more vigorous closer to the sunspot, or the MMFs are initially carried outwards partly by the momentum of the Evershed flow, which has speeds of $1-2 \mathrm{~km} \mathrm{~s}^{-1}$ in the canopy of a sunspot (Solanki et al. 1994) before aerodynamic drag slows them down to the ambient speed of the moat flow. In the first case we expect the speed of the MMFs to decrease mainly as a function of distance to the sunspot. In the latter case we expect the MMF speed to decrease as a function of distance from the point at which the MMF was formed. The results of our study clearly favour the second hypothesis, which is consistent with the U-loop model of MMF pairs proposed by Zhang et al. (2003).

\section{Discussions and conclusions}

Zhang et al. (2003) reported that MMF bipoles are not randomly oriented (see also Yurchyshyn et al. 2001), but rather that the member of an MMF pair further from the sunspot has the polarity of the parent sunspot in $85 \%$ of the cases, the orientations of MMF pairs are further associated with the twist of the sunspot superpenumbra. This supports the picture that MMF pairs are parts of U-loops "hanging" below a sunspot's superpenumbral 

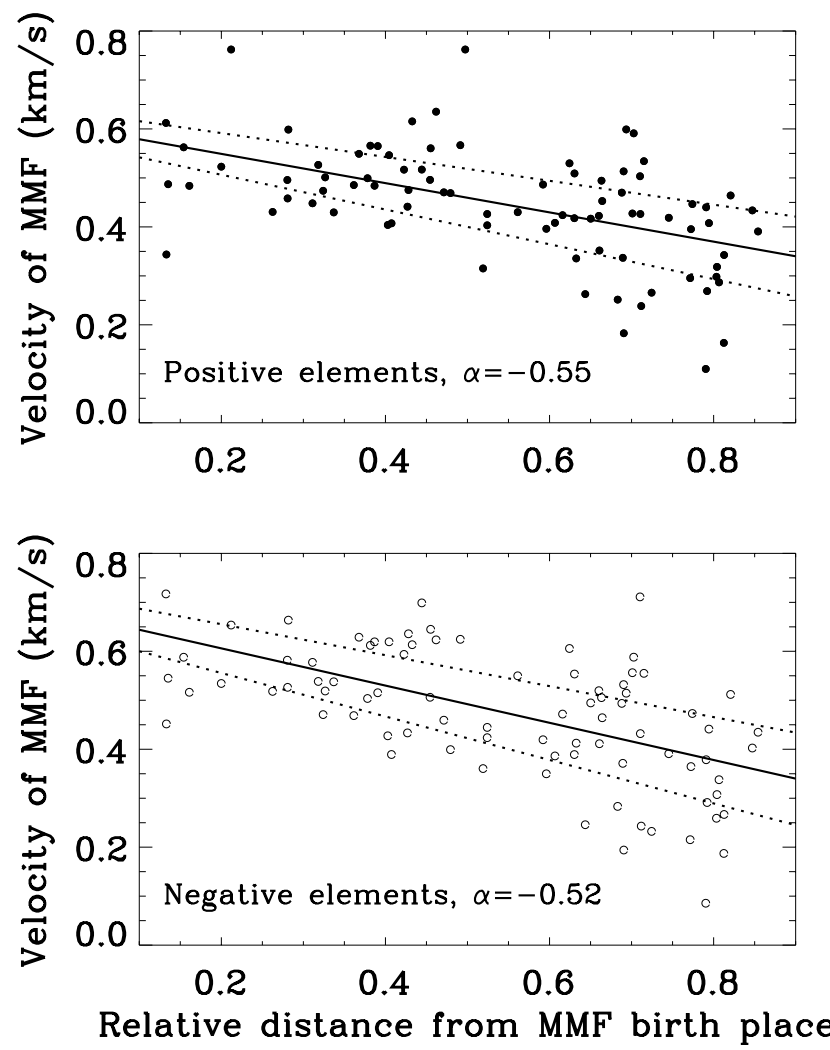

Fig. 8. The horizontal velocity of MMF elements is plotted versus relative distance from MMF birth place. Otherwise the figure is similar to Fig. 7.

canopy (see Fig. 9 of Zhang et al. 2003). Additionally, more MMF pairs are seen in the direction of the opposite polarity pore/sunspot in AR 8375, a direction in which the canopy is expected to lie particularly low.

In the model of Zhang et al. (2003) the Evershed flow plays an important role in the formation of MMF pairs. At the edge of the penumbra this supporting force disappears and a sufficiently dense and massive packet of Evershed gas cannot be supported by the flux-tube field any more. This gas then sinks, taking the magnetic field with it. In this way a U-loop is created near the penumbral edge, so MMF pairs first appear just outside the penumbrae (see Fig. 2). Alternatively, the flux tube can sink already within the penumbra, if it gets deformed downward. Such a deformation leads to a downflow, which makes this part of the tube more dense, making it bend down more, and so on, leading to an instability (see Schlichenmaier 2002), so that basically the same mechanism can lead to the production of MMFs outside the penumbra, or inside it (Sainz Dalda \& Martínez Pillet 2005; Ravindra 2006; Zhang et al. 2007). We expect the footpoint with opposite polarity to that of the sunspot to show signs of this downflowing material. Since the gas density increases rapidly with depth we do not expect a corresponding upflow in the other footpoint. Figures 4 and 5 show two examples of MMF pairs in which the negative element (close to the sunspot with positive polarity) has a downflow. Figure 6 confirms that this is a general phenomenon. The majority of the negative elements show a significant downflow compared to the positive polarity.

This downflow may be related to the isolated downflow seen just outside a sunspot by Börner \& Kneer (1992) and may partly explain why the Evershed velocity in the superpenumbra does not increase with distance from the sunspot, although the increasing canopy height implies that the mass flux must decrease
(Solanki et al. 1994, 1999): a part of the mass drains down into MMFs. The rather low MMF downflow velocities suggest that the field in the MMF pairs is heavily inclined to the vertical. This property is shared with the field lines found to submerge at the edge of the penumbra by Westendorp Plaza et al. (1997) and Mathew et al. (2002). By analogy to the MMF pairs we therefore expect such field lines also to eventually rejoin the magnetic canopy. This picture is supported by the simulations of Schlichenmaier (2002). Recently, Cabrera Solana et al. (2006) provide strong evidence that at least some MMFs are the continuation of the penumbral Evershed flow into the moat, this gives further support to the earlier results of Zhang et al. (2003) about the magnetic connection between MMFs and the penumbra. Note that in other models of MMFs, that do not involve U-loops from the canopy, a downflow predominantly in the MMF pair member closer to the spot is less easy to accommodate in a natural way.

The observation that the MMFs initially move faster early in their life before slowing down (see Fig. 8), suggests that although the moat flow may be the prime driver of older MMFs, other mechanisms, e.g. the Evershed flow, help drive younger MMFs. Changes in the moat flow cannot be the main cause of this deceleration, since the MMFs' velocity correlates poorly with the distance from the sunspot (Fig. 7). These results further confirm our MMF model (Zhang et al. 2003) that MMF pairs, i.e. type I MMFs are formed when the field lines in a small part of the magnetic canopy dip down to produce a U-loop.

Besides looking for further evidence to test models of MMFs we have also checked if the properties of MMFs change between young and old sunspots. The second main result we find is that the lifetime of MMFs around young sunspots is quite a bit longer than around older ones.

Also, the downflows in MMFs are more pronounced around younger sunspots. Specifically, for the young AR studied here nearly all MMF elements with polarity opposite to the sunspot show a significant redshift compared to the other elements. Of course, many more sunspots at different stages of their development need to be studied before we can be certain that MMF properties do depend on evolution stages of sunspots.

Acknowledgements. We thank the referee, Dr. Jose Carlos del Toro Iniesta, for the helpful comments and constructive suggestions to this manuscript. The authors are indebted to the SOHO/MDI team for providing the employed data. SOHO is a mission of international cooperation between ESA and NASA. J.Z. was supported by the National Natural Science Foundations of China (G10573025 and 40674081), the CAS Project KJCX2-YW-T04, the National Basic Research Program of China under grant G2006CB806303, and the cooperation agreement between the Chinese Academy of Sciences and the Max-Planck Society.

\section{References}

Börner, P., \& Kneer, F. 1992, A\&A, 259, 307

Borrero, J. M., Lagg, A., Solanki, S. K., \& Collados, M. 2005, A\&A, 436, 333 Brickhouse, N. S., \& LaBonta, B. J. 1988, Sol. Phys., 115, 43

Bumba, V. 1960, Izv. Crim. Astrophys. Obs., 23, 253

Cabrera Solana, D., Bellot Rubio, L. R., Beck, C., \& del Toro Iniesta, J. C. 2006, ApJ, 649, L41

del Toro Iniesta, J. C., Bellot Rubio, L. R., \& Collados, M. 2001, ApJ, 549, L139 Evershed, J. 1909, MNRAS, 69, 454

Hagenaar, H. J., \& Shine, R. A. 2005, ApJ, 635, 659

Harvey, K., \& Harvey, J. 1973, Sol. Phys., 28, 61

Hirzberger, J., \& Kneer, F. 2001, A\&A, 378, 1078

Lee, J. W. 1992, Sol. Phys., 139, 267

Mathew, S. K., Solanki, S. K., Lagg, A., et al. 2002, in Poster Proc. 1st Potsdam Thinkship on Sunspot \& Starspots, ed K. Strassmeier, AIP, 117

Muller, R., \& Mena, B. 1987, Sol. Phys., 112, 295

Muller, R. 1992, in NATO ASI Proc. 375: Sunspots. Theory and Observations, ed. J. H. Thomas, \& N. O. Weiss (Dordrecht: Kluwer), 175 
Ravindra, B. 2006, Sol. Phys., 237, 297

Ryutova, M., Shine, R., Title, A., \& Sakai, J. I. 1998, ApJ, 492, 402

Sainz Dalda, A., \& Martínez Pillet, V. 2005, ApJ, 632, 1176

Scherrer, P. H., Bogart, R. S., Bush, R. I., et al. 1995, Sol. Phys., 162, 129

Schlichenmaier, R. 2002, Astron. Nachr., 323, 342

Schlichenmaier, R., \& Schmidt, W. 2000, A\&A, 358, 1122

Sheeley, N. R. Jr. 1969, Sol. Phys., 9, 347

Sheeley, N. R. Jr. 1972, Sol. Phys., 25, 98

Shine, R., \& Title, A. 2001, Encyclopedia of Astronomy \& Astrophysics, 4, 3209

Solanki, S. K. 2003, A\&ARv, 11, 153

Solanki, S. K., Montavon, C. A. P., \& Livingston, W. 1994, A\&A, 283, 221

Solanki, S. K., Finsterle, W., Rüedi, I., \& Livingston, W. 1999, A\&A, 347, L27

Spruit, H. C., Title, A. M., \& van Ballegooijen, A. A. 1987, Sol. Phys., 110, 115

Thomas, J. H. 1994, in Solar Surface Magnetism, ed. R. J. Rutten, \& C. J. Schrijver (Dordrecht: Kluwer), 219

Tritschler, A., Schlichenmaier, R., Bellot Rubio, L. R., et al. 2004, A\&A, 415, 717
Vrabec, D. 1971, in Solar Magnetic Fields, ed. R. Howard (Dordrecht: Reidel), IAU Symp., 43, 329

Weiss, N. O., Thomas, J. H., Brummell, N. H., \& Tobial, S. M. 2004, ApJ, 600, 1073

Westendorp Plaza, C., del Toro Iniesta, J. C., Ruiz Cobo, B., et al. 1997, Nature, 389,47

Westendorp Plaza, C., del Toro Iniesta, J. C., Ruiz Cobo, B., et al. 2001, ApJ, 547,1130

Wiehr, E. 1995, A\&A, 298, L17

Wilson, P. R. 1973, Sol. Phys., 32, 435

Wilson, P. R. 1986, Sol. Phys., 106, 1

Yurchyshyn, V. B., Wang, H., \& Goode, P. R. 2001, ApJ, 550, 470

Zhang, J., Wang, J., Wang, H., \& Zirin, H. 1998, A\&A, 335, 341

Zhang, J., Solanki, S. K., \& Wang, J. 2003, A\&A, 399, 755

Zhang, J., Solanki, S. K., \& Woch, J. 2007, A\&A, submitted 